\title{
Lasing in Ni Nanodisk Arrays
}

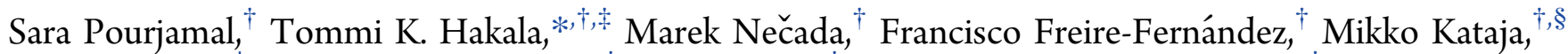
Heikki Rekola, ${ }^{\perp}$ Jani-Petri Martikainen, ${ }^{\dagger}$ Päivi Törmä, ${ }^{\dagger}$ a and Sebastiaan van Dijken ${ }^{*}{ }^{\dagger}$ (1)

${ }^{\dagger}$ Department of Applied Physics, Aalto University School of Science, FI-00076 Aalto, Finland

${ }^{*}$ Institute of Photonics, University of Eastern Finland, FI-80101 Joensuu, Finland

${ }^{\S}$ Institut de Ciència de Materials de Barcelona (ICMAB-CSIC), Campus de la UAB, Bellaterra 08193, Catalonia, Spain

${ }^{\perp}$ Smart Photonic Materials, Faculty of Engineering and Natural Sciences, Tampere University, FI-33101 Tampere, Finland

\section{Supporting Information}

ABSTRACT: We report on lasing at visible wavelengths in arrays of ferromagnetic $\mathrm{Ni}$ nanodisks overlaid with an organic gain medium. We demonstrate that by placing an organic gain material within the mode volume of the plasmonic nanoparticles both the radiative and, in particular, the high ohmic losses of Ni nanodisk resonances can be compensated. Under increasing pump fluence, the systems exhibit a transition from lattice-modified spontaneous emission to lasing, the latter being characterized by

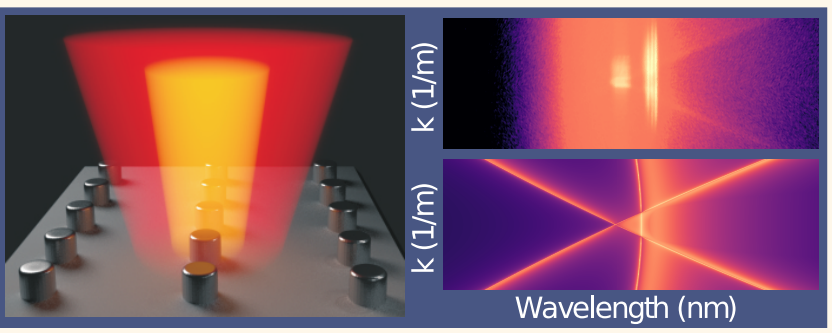
highly directional and sub-nanometer line width emission.

By breaking the symmetry of the array, we observe tunable multimode lasing at two wavelengths corresponding to the particle periodicity along the two principal directions of the lattice. Our results are relevant for loss-compensated magnetoplasmonic devices and topological photonics.

KEYWORDS: plasmonics, nanolasing, Ni nanodisk array, surface lattice resonance, loss-compensated magnetoplasmonics

$\mathrm{P}$ lasmonic resonators and cavities provide small mode volumes and ultrafast light-matter interactions at the nanoscale. Interactions between emitters and plasmonic modes have been studied in both weak and strong coupling regimes. ${ }^{1,2}$ Theoretical and experimental investigations on lasing in plasmonic systems have demonstrated the feasibility of compensating losses typical for metallic nanostructures ${ }^{3-23}$ and providing ultrafast operation speeds. ${ }^{8,24}$ Lasing as a losscompensation mechanism would be particularly attractive for magnetoplasmonics, which enables active light manipulation at the nanoscale via field-controlled breaking of time-reversal symmetry $^{25}$ and versatile design of magneto-optical responses. $^{26}$ Potential applications of magnetoplasmonic nanostructures include nonreciprocal optical devices, ${ }^{27}$ label-free biosensors, ${ }^{28,29}$ metrology, ${ }^{30}$ and ultrafast all-optical magnetic switching. ${ }^{31}$ However, ohmic losses in ferromagnetic metals are considerably larger than those in noble metals, and the narrowing of intrinsically broad resonances via a transition to lasing has not been demonstrated yet.

Periodic arrays of metallic nanoparticles support collective surface lattice resonances (SLRs) that originate from radiative coupling of lossy single particle plasmon resonances with lowloss diffracted orders (DOs) of the lattice..$^{32-37}$ In optical transmission spectra, the DOs of an array appear as maxima at $\lambda \approx n p$, where $n$ is the refractive index of the surrounding medium and $p$ is the particle periodicity. Hybridized Fano-like SLR modes reduce the signal, causing minimum transmission at a wavelength that deviates from the DO. ${ }^{33-37}$ Despite the plasmonic component, SLRs in arrays of noble metal nanodisks have particularly narrow line widths that can be utilized in lasing ${ }^{13-17,19,20,22}$ and Bose-Einstein condensation. ${ }^{38}$ Recently, it was demonstrated that collective SLR modes can be excited also in arrays of higher-loss ferromagnetic nanoparticles. ${ }^{39,40}$

Here, we report on lasing in a lattice of ferromagnetic nanodisks overlaid with optically pumped organic Rhodamine 6G (R6G) dye solution. Reduced line widths provided by the SLRs together with a carefully optimized lattice geometry and gain medium produce lasing at visible wavelengths, despite the broad plasmonic resonances of the individual nanodisks. Lasing is characterized by a highly directional and nonlinear increase of sub-nanometer line width emission by more than 2 orders of magnitude. Within the limits set by the gain profile of R6G, the lasing wavelength can be tuned by varying the particle periodicity. In rectangular arrays, we observe lasing at two wavelengths corresponding to $\lambda \approx n \times p_{i}$ for different particle periodicities $\left(p_{x}\right.$ and $\left.p_{y}\right)$ along the two principle axes of the lattice.

Received: February 4, 2019

Accepted: April 11, 2019

Published: April 11, 2019 
(a) Pump, $500 \mathrm{~nm}, 100 \mathrm{fs}, 1 \mathrm{kHz}$

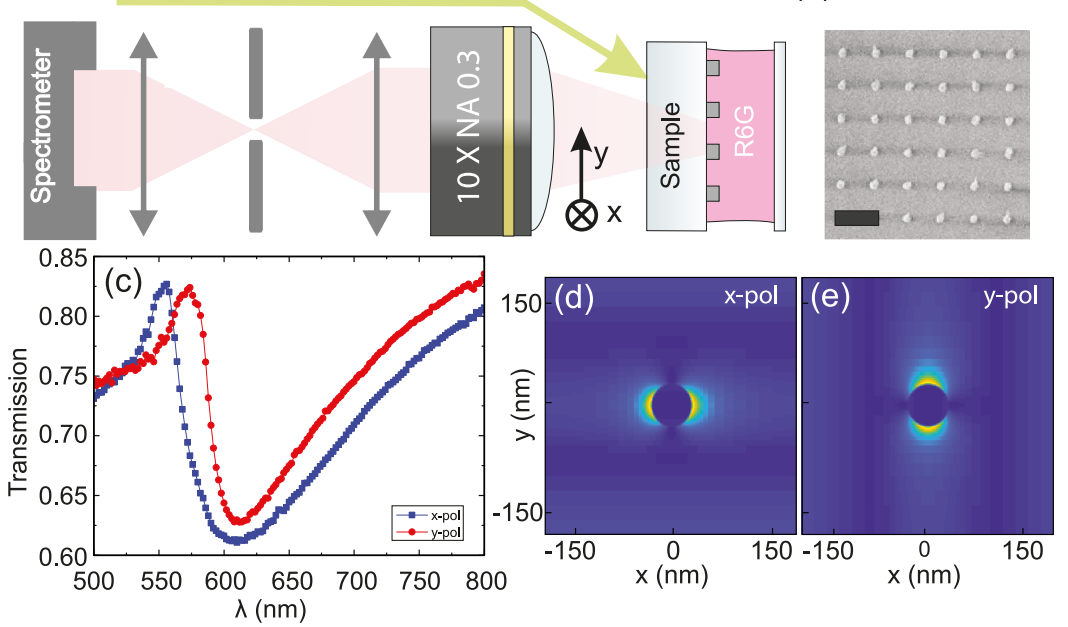

Figure 1. (a) Schematic of the measurement setup. Emission spectra are measured by focusing the back focal plane of the objective lens to the entrance slit of the spectrometer. The gain medium is pumped by $x$-polarized $100 \mathrm{fs}$ laser pulses with a wavelength of $500 \mathrm{~nm}$ and a 1 $\mathrm{kHz}$ repetition rate. The gain medium is inserted between the substrate with Ni nanodisk arrays and a cover glass. (b) Scanning electron microscopy image of the $380 \mathrm{~nm} \times 380 \mathrm{~nm}$ array. In all experiments, the diameter and height of the Ni nanodisks are $60 \mathrm{~nm}$. The scale bar corresponds to $500 \mathrm{~nm}$. (c) Experimental transmission curves for the Ni nanodisk array with $p_{x}=380 \mathrm{~nm}$ and $p_{y}=370 \mathrm{~nm}$. Data for incident polarization along the $x$ and $y$ directions of the array are shown. (d,e) Finite-difference time-domain simulations of near-field distributions in the same array. The simulations are performed for $x$ - and $y$-polarized plane-wave excitation at the SLR wavelength.

\section{RESULTS AND DISCUSSION}

In our study, we used $\mathrm{Ni}$ as a ferromagnetic plasmonic material. We fabricated various arrays of Ni nanodisks on glass substrates using electron-beam evaporation and lift-off in an electron-beam lithography process. The nominal diameter and height of the nanodisks were $60 \mathrm{~nm}$, and the size of the total array was $300 \mu \mathrm{m} \times 300 \mu \mathrm{m}$. As a reference, we considered a square array with $p_{x}=p_{y}=380 \mathrm{~nm}$. In rectangular arrays with broken symmetry, $p_{x}$ was kept constant and $p_{y}$ was varied from 370 to $390 \mathrm{~nm}$ in $5 \mathrm{~nm}$ steps. The gain medium consisting of $35 \mathrm{mM} \mathrm{R6G}$ in 1:2 dimethyl sulfoxide (DMSO)/benzyl alcohol (BA) was inserted between the substrate with $\mathrm{Ni}$ nanodisk arrays and a cover glass.

A schematic of the measurement setup including excitation and detection lines is depicted in Figure 1a. The sample was excited by $100 \mathrm{fs}$ laser pulses with a wavelength of $500 \mathrm{~nm}$ at a $1 \mathrm{kHz}$ repetition rate and from a $45^{\circ}$ angle. The diameter of the pump spot was approximately $100 \mu \mathrm{m}$. Emitted light from the sample was collected with a $10 \times 0.3 \mathrm{NA}$ objective lens. The back focal plane of the objective lens was focused to the entrance slit of a spectrometer. The long axis of the slit was aligned along the $y$-axis of the sample. From 2D intensity data collected by the CCD camera of the spectrometer, the wavelength and in-plane $k_{y}$ vector were calculated using $k_{y}=$ $k_{0} \sin (\theta) .{ }^{19}$ Here $k_{0}=2 \pi / \lambda$ and $\theta$ is the angle with respect to the sample normal. We note that we did not apply a magnetic field during the lasing experiments. A scanning electron microscopy image of the square $380 \mathrm{~nm} \times 380 \mathrm{~nm}$ reference array is shown in Figure $1 \mathrm{~b}$.

Figure 1c shows experimental transmission curves of the $\mathrm{Ni}$ nanodisk array with $p_{x}=380 \mathrm{~nm}$ and $p_{y}=370 \mathrm{~nm}$ for incident polarization along $x$ and $y$ (see Supporting Information, Figure S1 for measurements on other arrays). The intensity maxima are close to the DOs of the lattice. Because the DO wavelength depends on the particle periodicity perpendicular to the polarization axis, the transmission curve for $x$-polarized light is blue-shifted with respect to the spectrum measured with $y$ - polarization. Coupling of a narrow DO to a broad localized surface plasmon resonance (LSPR) in the $\mathrm{Ni}$ nanodisks produces a collective SLR mode. ${ }^{39}$ The SLR wavelength (minimum transmission in Figure 1c) corresponds to the wavelength where $1 / \alpha-S$ is $0 .{ }^{34,35}$ Here, $S$ is the so-called array factor and $\alpha$ is the polarizability of a single Ni nanodisk. Damping of the SLR mode depends sensitively on the imaginary part of $1 / \alpha$. Consequently, noble metal nanodisks with large polarizability produce narrow SLR modes with a line width of $<10 \mathrm{~nm}$ when ordered into periodic arrays. ${ }^{32-37}$ Because of larger ohmic losses in $\mathrm{Ni}$ (i.e., small $\alpha$ ), the SLRs in our plasmonic arrays are much broader $(>100 \mathrm{~nm}$; see Figure 1c). Finite-difference time-domain (FDTD) simulations of the $380 \mathrm{~nm} \times 370 \mathrm{~nm}$ Ni nanodisk array at the SLR wavelengths (Figure 1d,e) show intense electric near fields in the particle plane, confirming the plasmonic character of this collective mode. Next, we demonstrate that despite the large line width of SLR excitations, it is possible to realize lasing in $\mathrm{Ni}$ nanostructures.

Figure 2a,b shows the $k_{y}$ and wavelength-resolved emission from the sample with a $380 \mathrm{~nm} \times 380 \mathrm{~nm} \mathrm{Ni}$ nanodisk array for a pump fluence below and above the lasing threshold $\left(P_{\text {th }} \approx\right.$ $3.3 \mathrm{~mJ} \mathrm{~cm}^{-2}$ ). Below the threshold, the emission consists of two contributions. The first contribution has no angular dependence and originates from the R6G molecules. Because the molecules are spatially far away from the nanoparticles (and outside the SLR mode volume), they do not emit to the SLR mode. Their emission spectrum has a line width of $\sim 60$ $\mathrm{nm}$, which is the same as that for the R6G dye solution in the absence of the nanodisk array. The second emission contribution follows the $\langle+1,0\rangle$ and $\langle-1,0\rangle$ DOs of the array (crossed dashed lines) and, thus, depicts spontaneous emission of the molecules to the SLR modes. As expected from $\lambda=n \times$ $p_{i}$ and the transmission curves of Figure 1c, the DOs related to the periodicity $p_{y}$ cross at $\lambda=578 \mathrm{~nm}$ if $p_{y}=380 \mathrm{~nm}$ and $n=$ 1.52. At a higher pump fluence of $1.3 P_{\mathrm{th}}$, we observe an intense single emission peak at $\lambda \approx 580 \mathrm{~nm}$ with a narrow line width of 

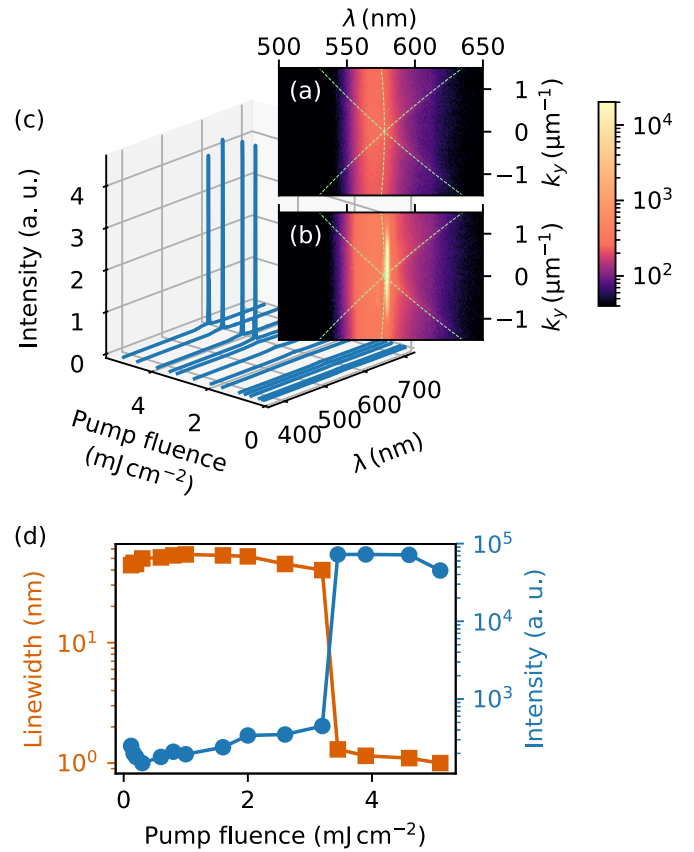

Figure 2. Angle and wavelength resolved emission of a symmetric $380 \mathrm{~nm} \times 380 \mathrm{~nm} \mathrm{Ni}$ nanodisk array below (a) and above (b) the lasing threshold. The dashed lines indicate the DOs of the array. Because the momentum direction $k_{y}$ is monitored here, the $\langle+1,0\rangle$ and $\langle-1,0\rangle$ DOs related to the periodicity $p_{y}$ appear as a cross feature, whereas the one related to $p_{x}($ at $578 \mathrm{~nm}$ ) has a parabolic shape. The former is sometimes called TE and the latter TM mode in the literature. ${ }^{2}$ (c) Emission intensity at $k_{y}=0$ as a function of pump fluence. (d) Line width (squares) and intensity (circles) of the emission peak showing an abrupt nonlinear change of these parameters at a threshold pump fluence $P_{\text {th }} \approx 3.3 \mathrm{~mJ} \mathrm{~cm}^{-2}$.

$<1 \mathrm{~nm}$ and a small beam divergence of $5.7^{\circ}$. The lasing peak is slightly red-shifted from the DOs to a wavelength where the R6G dye solution can emit to the SLR modes of the $\mathrm{Ni}$ nanodisk array. The transition from spontaneous emission to lasing is manifested as an abrupt change in the emission spectrum (Figure 2c). Figure $2 \mathrm{~d}$ summarizes the variation of the emission intensity and line width with increasing pump fluence. Most notably, we measure a strongly nonlinear increase of the emission intensity from $\sim 2 \times 10^{2}$ to $\sim 10^{5}$ if the pump fluence is enhanced from 3.2 to $3.45 \mathrm{~mJ} \mathrm{~cm}^{-2}$. Simultaneously, the line width of the emission peak decreases from $\sim 60$ to $<1 \mathrm{~nm}$. We note that the lasing peak line width of our Ni system is similar to those obtained with $2 \mathrm{D}$ arrays of $\mathrm{Au}, \mathrm{Ag}$, or $\mathrm{Al}$ nanoparticles (values range from 0.1 to $1.5 \mathrm{~nm}$ in refs $13,18,19,21$, and 22). Thus, despite much larger losses in Ni nanodisk arrays (SLR line width $>100 \mathrm{~nm}$ compared to 4$10 \mathrm{~nm}$ in noble metal lattices), intense and narrow line width lasing is still attained if enough gain is provided. This finding demonstrates that lasing can be used as a powerful tool to circumvent losses in magnetoplasmonic systems.

Next, we break the symmetry of the Ni nanodisk array by keeping $p_{x}$ constant and increasing $p_{y}$ from 370 to $390 \mathrm{~nm}$ in 5 $\mathrm{nm}$ steps. Figure 3 shows emission spectra of these samples for

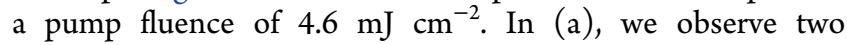
emission maxima, one at the same wavelength, $\lambda \approx 580 \mathrm{~nm}$, as that for the square array (see Figure $3 \mathrm{c}$ ) and the other at $\lambda \approx$ $565 \mathrm{~nm}$. Threshold behavior and line width imply lasing action for both peaks. We note that the maximum at $565 \mathrm{~nm}$ is $15 \mathrm{~nm}$ blue-shifted from the other lasing peak. We associate this

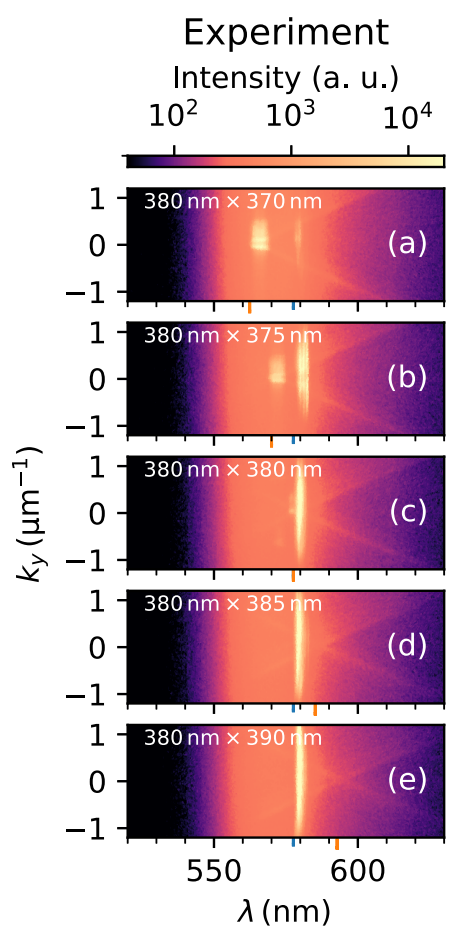

Theory

Lowest singular value 1

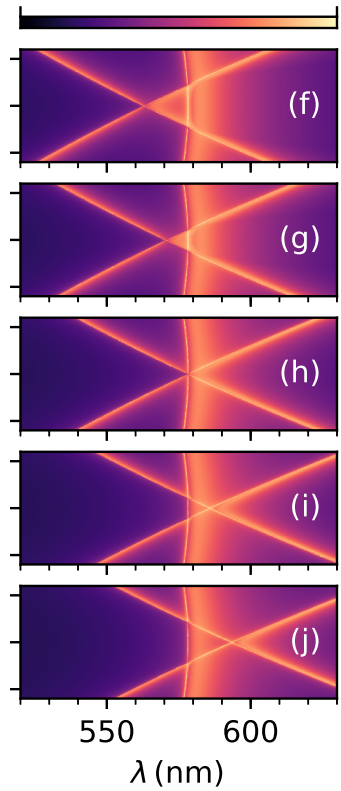

Figure 3. $(\mathrm{a}-\mathrm{e})$ Angle- and wavelength-resolved emission data for samples having $p_{x}=380 \mathrm{~nm}$ and $p_{y}$ ranging from 370 to $390 \mathrm{~nm}$ at a pump fluence of $4.6 \mathrm{~mJ} \mathrm{~cm}$. The red ticks label the crossing wavelength of the $\langle-1,0\rangle$ and $\langle+1,0\rangle$ diffracted orders related to the periodicity $p_{y}$ of the array. $(\mathrm{f}-\mathrm{j})$ Dispersions of respective ideal infinite arrays computed using the $T$-matrix method for $E$ in-plane modes. Dispersion bands of the array are characterized by singular values of the underlying scattering problem (1) reaching near 0.

emission with the reduced particle periodicity along the $y$ direction. The expected $15 \mathrm{~nm}$ blue-shift based on $\Delta \lambda=n \times$ $\Delta p_{y}$ supports this argument, as well as the FDTD simulations of Supporting Information, Figure S3. The emission maximum at $580 \mathrm{~nm}$ is associated with the larger periodicity of the rectangular Ni nanodisk array along the $x$ direction.

In agreement with the dependence of the two lasing peaks on particle periodicity, the lower-wavelength emission maximum red shifts when $p_{y}$ increases to $375 \mathrm{~nm}$, whereas the other peak remains fixed at $580 \mathrm{~nm}$ (Figure $3 \mathrm{~b}$ ). A further increase of $p_{y}$ to $380 \mathrm{~nm}$ results in a square nanodisk lattice, and consequently, the two emissions merge into one intense lasing peak (Figure $3 \mathrm{c}$ ). For $p_{y}>380 \mathrm{~nm}$, one would expect a second lasing peak to appear at $\lambda>580 \mathrm{~nm}$. As can be seen from the emission data in Figure 3d,e, this is not the case. We explain the absence of the anticipated second peak by a reduced overlap of the SLR mode with the wavelengthdependent gain profile of the R6G dye solution. At wavelengths corresponding to $p_{y}>380 \mathrm{~nm}$, the R6G gain is insufficient to compensate for the lossy SLR mode of the $\mathrm{Ni}$ nanodisk array, resulting in much weaker spontaneous emission instead of lasing. For $p_{y}<380 \mathrm{~nm}$, the spectrum of the R6G gain medium overlaps more with the energy of the $p_{y^{-}}$ related SLR mode, and consequently, multimode lasing is observed. Two-mode lasing has been observed previously for noble metals. ${ }^{19,21}$ Moreover, for $\mathrm{Al}$ nanoparticles, it has been shown that highly asymmetric $1 \mathrm{D}$ arrays do also lase. ${ }^{22}$

To understand the mode properties, we employ a multiplescattering $T$-matrix approach 


$$
M(\omega, \boldsymbol{k}) a_{\nu}(\omega, \boldsymbol{k})=0
$$

where $M(\omega, k)$ is a matrix depending on scattering and wave propagation properties of the array at a given frequency $\omega$ and $a_{\nu}(\omega, k)$ is a vector of coefficients describing multipole nanoparticle excitations of a given mode. The problem (1) has a nontrivial solution; that is, a mode exists if the matrix $M(\omega$, $k$ ) has a 0 singular value (SV). Therefore, to find modes supported by the array, we scan $\omega, k$ space to search for SV minima of $M(\omega, k)$. Due to losses, SV minima are not exactly 0 for real $\omega, k$, but near 0 SVs nevertheless provide valuable information about the dispersion of the array. Figure $3 \mathrm{f}-\mathrm{j}$ illustrates calculated modes with the lowest SVs of $M(\omega, k)$, resembling the experimental data of Figure $3 \mathrm{a}-\mathrm{e}$. The Materials and Methods section provides more details on the numerical model.

Finally, we analyze the polarization properties of the lasing modes in our Ni nanodisk samples with a R6G gain medium. Experimentally, this was done by placing a polarizer between the sample and spectrometer to determine the emission intensity with polarization along the $x$ and $y$ directions of the arrays. Figure $4 \mathrm{a}-\mathrm{e}$ presents emission spectra for both

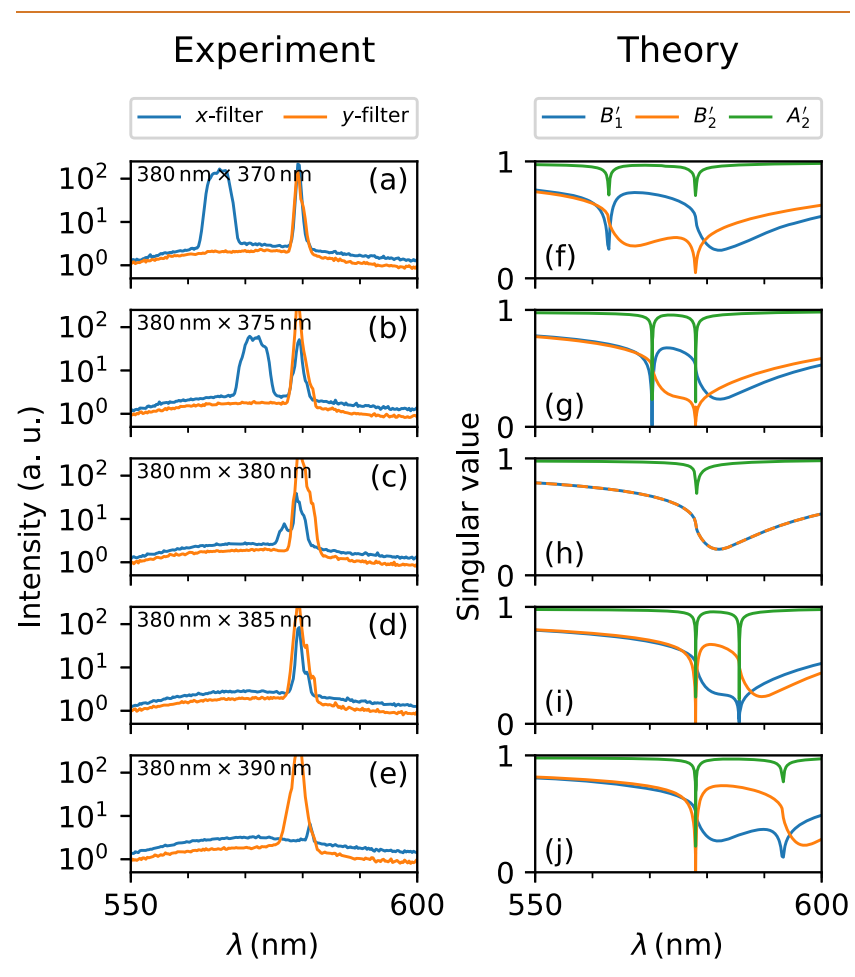

Figure 4. $(\mathrm{a}-\mathrm{e})$ Emission spectra at $k_{y}=0$ for samples having $p_{x}=$ $380 \mathrm{~nm}$ and $p_{y}$ ranging from 370 to $390 \mathrm{~nm}$. The pump fluence is $4.6 \mathrm{~mJ} \mathrm{~cm}^{-2}$. ( $\left.\mathrm{f}-\mathrm{j}\right)$ Calculated singular values for arrays with the same particle periodicities. The blue and orange colors correspond to $x$ - and $y$-polarized dipolar modes $\left(B_{1,2}^{\prime}\right)$, respectively, and the green color corresponds to a quadrupolar mode $\left(A_{2}^{\prime}\right)$.

polarization states at $k_{y}=0$ and a pump fluence of $4.6 \mathrm{~mJ}$ $\mathrm{cm}^{-2}$. In (a), the low wavelength emission related to $p_{y}$ is $x$ polarized, whereas the $p_{x}$-related lasing peak at $580 \mathrm{~nm}$ exhibits both polarizations. We note that the $x$-polarization of this peak disappears at higher pump fluence (see Supporting Information, Figures S4 and S5). We observe similar behavior in (b), with a red shift in the lower wavelength lasing peak because of larger $p_{y}$. For the square array (c), we observe lasing at a single wavelength due to mode degeneracy. In (d), lasing takes place at the wavelength of the $p_{x}$-related SLR mode only. The lasing peak in this emission spectrum exhibits both polarizations. Finally, in (e), single mode lasing with reduced $x$-polarization is measured.

The polarization properties are also studied by the $T$-matrix method, which can uncover both $x$ - and $y$-polarized dipolar as well as quadrupolar contributions at each wavelength. Figure $4 \mathrm{f}-\mathrm{j}$ summarizes the results for the different $\mathrm{Ni}$ nanodisk arrays. In (f), SV minima are calculated for two dipolar modes corresponding to the experimentally observed wavelengths in (a) and polarizations at high pump fluence. The same applies for $(\mathrm{g})$. In (h), the square array exhibits degeneracy of the dipolar modes. This raises the question whether the experimental observations in (c) are caused by lasing action of two perpendicular dipolar modes or a quadrupolar mode. Finally, in (i) and ( $j)$, we note that the model predicts a large quadrupolar weight at the measured lasing wavelength in (d) and (e). In (d), the experimental lasing peak exhibits both polarizations, and hence, the mode is indeed quadrupolar. In contrast, lasing in (e) is almost purely $y$-polarized, suggesting dipolar mode lasing. To rationalize this, we point out that $a$ priori predictions of preferred lasing modes are difficult because mode dynamics, mode competition at available gain, and mode Q-factors all play a role. Furthermore, we observe a dependence of lasing behavior on pump fluence (see Supporting Information, Figure S4 for a complete set of emission spectra at different pump fluence). A more detailed understanding of mode competition in $\mathrm{Ni}$ nanodisk arrays requires further studies.

\section{CONCLUSIONS}

In summary, we demonstrate lasing in a high-loss ferromagnetic plasmonic system. In rectangular Ni nanodisk arrays, we observe multimode lasing and the coexistence of dipolar and quadrupolar modes. The results of this paper can be used for incorporating gain into magnetoplasmonic devices and realizing concepts for topological photonics. Notably, topological lasing has been demonstrated recently. ${ }^{42-44}$ In topological photonics, ${ }^{45-48}$ most lattice systems are based on nearest or next-nearest neighbor coupling via overlapping optical nearfields. Our Ni nanodisk arrays represent a radiatively coupled system where long-range couplings produce collective SLR modes. The symmetry properties of the array dictate the existence of energy degenerate modes at high-symmetry points of the Brillouin zone, ${ }^{41}$ for which the lifting of the degeneracy by a symmetry breaking mechanism can lead to topological features. The magnetic moment of nanodisks in a ferromagnetic array can be exploited for time-reversal symmetry breaking in such lattices. The $\mathrm{Ni}$ nanodisk arrays discussed in this paper show a magnetic circular dichroism effect of up to $0.5 \%$ in a saturating magnetic field of $150 \mathrm{mT}^{49}$

\section{MATERIALS AND METHODS}

Sample Preparation. We fabricated the samples on glass substrates using electron-beam lithography. After a poly(methyl methacrylate) (PMMA) layer was spin-coated and baked at $180{ }^{\circ} \mathrm{C}$ for $1 \mathrm{~min}$, the pattern was defined by exposing the resist layer to the electron beam. We developed the PMMA in a 1:3 methyl isobutyl ketone/isopropyl alcohol solution. Ni nanodisk arrays were fabricated by e-beam evaporation of a $60 \mathrm{~nm}$ thick film, followed by lift-off. After being patterned, the nanodisk arrays were covered by $2 \mathrm{~nm} \mathrm{Al}_{2} \mathrm{O}_{3}$ 
using atomic layer deposition to protect $\mathrm{Ni}$ from degrading when contacted by R6G molecules.

Sample Characterization. We measured optical transmission curves using a spectrometer with a broad-band supercontinuum laser. For lasing measurements, the R6G gain medium was inserted between the glass substrate with $\mathrm{Ni}$ nanodisk arrays and a cover glass. The gain medium was pumped with a femtosecond laser $\left(45^{\circ}\right.$ incident angle, $500 \mathrm{~nm}$ center wavelength, $100 \mathrm{fs}$ pulse width, $1 \mathrm{kHz}$ repetition rate, $x$-polarization). Emission spectra were recorded as a function of wavelength and in-plane $k_{y}$-vector by focusing the back focal plane of a $10 \times 0.3 \mathrm{NA}$ objective onto the slit of a spectrometer (Figure 1a). The lasing peaks were measured with three settings of the output polarization filter ( $x$ filter, $y$ filter, no filter).

Simulations. The dispersion calculations in Figures $3 \mathrm{f}-\mathrm{j}$ and $4 \mathrm{f}-\mathrm{j}$ were performed using a multiple-scattering $T$-matrix approach. Here, we briefly introduce the model. A more detailed description can be found in the Supporting Information of ref 41.

The scattering properties of a single nanoparticle are first described in terms of vector spherical wave functions (VSWFs), giving the Tmatrix of the nanoparticle at a given frequency. In the next step, electromagnetic interactions between nanoparticles are calculated as translation operators $S_{n, n^{\prime}}$, which describe how VSWFs with one coordinate origin (located inside the nanoparticle $n^{\prime}$ ) can be decomposed in a VSWF basis at another origin (corresponding to the location of nanoparticle $n$ ). This yields a set of linear equations describing the scattering properties of a system with many nanoparticles, which, in a per-particle matrix form, can be written as

$$
a_{n}-T_{n} \sum_{n^{\prime} \neq n} S_{n, n^{\prime}} a_{n^{\prime}}=T_{n} p_{\operatorname{ext}(n)}
$$

Here, the $p_{\text {ext }(n)}$ column vectors contain the VSWF expansion coefficients of the external electromagnetic radiation incident onto the $n$th nanoparticle, and $a_{n}$ is the vector of the unknown VSWF expansion coefficients of the scattered field created by the $n$th nanoparticle.

For periodic systems, the field expansion coefficients at different nanoparticle sites attain the Bloch waveform of $a_{i}=e^{i k \cdot \boldsymbol{R}_{i}} a$, where $\boldsymbol{R}_{i}$ are the lattice vectors and $k$ is the Bloch wave vector. If there is only one nanoparticle per unit cell and the $T$-matrices are the same for all particles, eq 2 can be rewritten as

$$
\begin{aligned}
& a_{i}-T \sum_{i^{\prime} \neq i} S_{i, i^{\prime}} i^{i \mathbf{k} \cdot\left(\boldsymbol{R}_{i^{\prime}}-\boldsymbol{R}_{i}\right)} a_{i^{\prime}}=T p_{\operatorname{ext}(i)} \\
& \text { Labeling } W=\sum_{i^{\prime} \neq i} S_{i, i^{\prime}} e^{i \boldsymbol{k} \cdot\left(\boldsymbol{R}_{i}^{\prime}-\boldsymbol{R}_{i}\right)}=\sum_{i^{\prime} \neq 0} S_{0, i^{\prime}} e^{i k \cdot \boldsymbol{R}_{i}^{\prime}} \text {, this gives } \\
& \quad(I-T W) a=T p_{\mathrm{ext}}
\end{aligned}
$$

where the only challenge remains in calculating the infinite lattice sums contained in the operator $W$, which in general diverge. One can work around this using Ewald-type summation techniques.

Equation 3 with a nonzero right-hand side describes the electromagnetic response of a periodic array on external periodic driving. Putting the right-hand side to 0 gives an equation for the electromagnetic modes supported by the array. The electromagnetic bands are then given for points $(\omega, \boldsymbol{k})$ in the frequencyquasimomentum space where the matrix $M(\omega, \boldsymbol{k}) \equiv I-T(\omega) W(\omega$, $k)$ is singular. In other words, the dispersion of the array can be calculated by finding a set of $(\omega, \boldsymbol{k})$ pairs for which $M(\omega, \boldsymbol{k})$ has a 0 singular value. This usually does not exactly happen for real $(\omega, k)$ in lossy systems like our Ni nanodisk arrays. However, the complex lossy bands are typically located near the real $(\omega, k)$ points for which some singular value of $M(\omega, k)$ reaches a small enough minimum.

Point-group symmetries of nanoparticle arrays provide a lot of $a$ priori information about the modes and enable us to simplify the calculations. ${ }^{50,51}$ All of the modes have to belong to the irreducible representations of the respective symmetry group. As a result, the $M(\omega, k=0)$ matrix can be written in block-diagonal form, where each block corresponds to one irreducible representation of the symmetry group. Singular values can then be searched for in each block separately. This is what we did to obtain the results of Figure $4 \mathrm{f}-\mathrm{j}$.
At the $\Gamma$ point (where the band edges responsible for lasing typically appear), our system exhibits $D_{2 h}$ symmetry (or even $D_{4 h}$ for the square array). The transformation properties of the irreducible representations under symmetry operations, encoded in the character tables, also determine which multipole excitations are allowed in each of the irreducible representations. In this way, we can determine which irreducible representations contain $x$ and $y$ dipolar nanoparticle polarizations or quadrupolar polarizations.

The $T$-matrix can be obtained using various numerical methods. For reliability reasons and due to the fact that the aspect ratio of our nanodisks is close to 1 , we used a spherical approximation and calculated the $T$-matrix analytically using the Lorenz-Mie solution, assuming a nanoparticle radius of $42 \mathrm{~nm}$, a homogeneous background medium with refractive index $n=1.52$, and nanoparticles made of $\mathrm{Ni}$ with interpolated optical properties from Johnson and Christy. ${ }^{52}$

Electric field distributions in the Ni nanodisk arrays were modeled using the Lumerical FDTD software.

\section{ASSOCIATED CONTENT}

\section{S Supporting Information}

The Supporting Information is available free of charge on the ACS Publications website at DOI: 10.1021/acsnano.9b01006.

Transmission curves for all Ni nanodisk arrays; FDTD lasing simulations; dependence of emission spectra on the laser pump fluence; polarization analysis of the lasing peaks (PDF)

\section{AUTHOR INFORMATION}

\section{Corresponding Authors}

*E-mail: tommi.hakala@uef.fi.

*E-mail: sebastiaan.van.dijken@aalto.fi.

ORCID

Päivi Törmä: 0000-0003-0979-9894

Sebastiaan van Dijken: 0000-0001-6372-2252

\section{Notes}

The authors declare no competing financial interest.

\section{ACKNOWLEDGMENTS}

This work was supported by the Academy of Finland under Project Nos. 303351, 307419, 318987, and 316857, by the European Research Council (ERC-2013-AdG-340748CODE), and by the Aalto Centre for Quantum Engineering. Lithography was performed at the Micronova Nanofabrication Centre, supported by Aalto University.

\section{REFERENCES}

(1) Törmä, P.; Barnes, W. L. Strong Coupling Between Surface Plasmon Polaritons and Emitters: A Review. Rep. Prog. Phys. 2015, 78, No. 013901.

(2) Moerland, R. J.; Hakala, T. K.; Martikainen, J.-P.; Rekola, H. T.; Törmä, P. In Quantum Plasmonics; Bozhevolnyi, S., Martin-Moreno, L., Garcia-Vidal, F., Eds.; Springer: Berlin, 2017; pp 121-150.

(3) Bergman, D. J.; Stockman, M. I. Surface Plasmon Amplification by Stimulated Emission of Radiation: Quantum Generation of Coherent Surface Plasmons in Nanosystems. Phys. Rev. Lett. 2003, 90, No. 027402.

(4) Hill, M. T.; Oei, Y.-S.; Smalbrugge, B.; Zhu, Y.; de Vries, T.; van Veldhoven, P. J.; van Otten, F. W. M.; Eijkemans, T. J.; Turkiewicz, J. P.; de Waardt, H.; Geluk, E. J.; Kwon, S.-H.; Lee, Y.-H.; Nötzel, R.; Smit, M. K. Lasing in Metallic-Coated Nanocavities. Nat. Photonics 2007, 1, 589-594.

(5) Stockman, M. I. Spasers Explained. Nat. Photonics 2008, 2, 327329.

(6) Zheludev, N. I.; Prosvirnin, S. L.; Papasimakis, N.; Fedotov, V. A. Lasing Spaser. Nat. Photonics 2008, 2, 351-354. 
(7) Noginov, M. A.; Zhu, G.; Belgrave, A. M.; Bakker, R.; Shalaev, V. M.; Narimanov, E. E.; Stout, S.; Herz, E.; Suteewong, T.; Wiesner, U. Demonstration of a Spaser-Based Nanolaser. Nature 2009, 460, $1110-1112$.

(8) Oulton, R. F.; Sorger, V. J.; Zentgraf, T.; Ma, R.-M.; Gladden, C.; Dai, L.; Bartal, G.; Zhang, X. Plasmon Lasers at Deep Subwavelength Scale. Nature 2009, 461, 629-632.

(9) Wuestner, S.; Pusch, A.; Tsakmakidis, K. L.; Hamm, J. M.; Hess, O. Overcoming Losses with Gain in a Negative Refractive Index Metamaterial. Phys. Rev. Lett. 2010, 105, 127401.

(10) Ma, R.-M.; Oulton, R. F.; Sorger, V. J.; Bartal, G.; Zhang, X. Room-Temperature Sub-Diffraction-Limited Plasmon Laser by Total Internal Reflection. Nat. Mater. 2011, 10, 110-113.

(11) Khajavikhan, M.; Simic, A.; Katz, M.; Lee, J. H.; Slutsky, B.; Mizrahi, A.; Lomakin, V.; Fainman, Y. Thresholdless Nanoscale Coaxial Lasers. Nature 2012, 482, 204-207.

(12) Lu, Y.-J.; Kim, J.; Chen, H.-Y.; Wu, C.; Dabidian, N.; Sanders, C. E.; Wang, C.-Y.; Lu, M.-Y.; Li, B.-H.; Qiu, X.; Chang, W.-H.; Chen, L.-J.; Shvets, G.; Shih, C.-K.; Gwo, S. Plasmonic Nanolaser Using Epitaxially Grown Silver Film. Science 2012, 337, 450-453.

(13) Zhou, W.; Dridi, M.; Suh, J. Y.; Kim, C. H.; Co, D. T.; Wasielewski, M. R.; Schatz, G. C.; Odom, T. W. Lasing Action in Strongly Coupled Plasmonic Nanocavity Arrays. Nat. Nanotechnol. 2013, 8, 506-511.

(14) van Beijnum, F.; van Veldhoven, P. J.; Geluk, E. J.; de Dood, M. J. A.; 't Hooft, G. W.; van Exter, M. P. Surface Plasmon Lasing Observed in Metal Hole Arrays. Phys. Rev. Lett. 2013, 110, 206802.

(15) Meng, X.; Kildishev, A. V.; Fujita, K.; Tanaka, K.; Shalaev, V. M. Wavelength-Tunable Spasing in the Visible. Nano Lett. 2013, 13, 4106-4112.

(16) Schokker, A. H.; Koenderink, A. F. Statistics of Randomized Plasmonic Lattice Lasers. ACS Photonics 2015, 2, 1289-1297.

(17) Dridi, M.; Schatz, G. C. Lasing Action in Periodic Arrays of Nanoparticles. J. Opt. Soc. Am. B 2015, 32, 818-823.

(18) Yang, A.; Hoang, T. B.; Dridi, M.; Deeb, C.; Mikkelsen, M. H.; Schatz, G. C.; Odom, T. W. Real-Time Tunable Lasing From Plasmonic Nanocavity Arrays. Nat. Commun. 2015, 6, 6939.

(19) Hakala, T. K.; Rekola, H. T.; Väkeväinen, A. I.; Martikainen, J.P.; Necada, M.; Moilanen, A. J.; Törmä, P. Lasing in Dark and Bright Modes of a Finite-Sized Plasmonic Lattice. Nat. Commun. 2017, 8, 13687.

(20) Ramezani, M.; Halpin, A.; Fernández-Domínguez, A. I.; Feist, J.; Rodriguez, S. R.-K.; Garcia-Vidal, F. J.; Gomez Rivas, J. PlasmonExciton-Polariton Lasing. Optica 2017, 4, 31-37.

(21) Wang, D.; Yang, A.; Wang, W.; Hua, Y.; Schaller, R. D.; Schatz, G. C.; Odom, T. W. Band-Edge Engineering for Controlled MultiModal Nanolasing in Plasmonic Superlattices. Nat. Nanotechnol. 2017, 12, 889-894.

(22) Rekola, H. T.; Hakala, T. K.; Törmä, P. One-Dimensional Plasmonic Nanoparticle Chain Lasers. ACS Photonics 2018, 5, 18221826.

(23) Wang, D.; Wang, W.; Knudson, M. P.; Schatz, G. C.; Odom, T. W. Structural Engineering in Plasmon Nanolasers. Chem. Rev. 2018, 118, 2865-2881.

(24) Daskalakis, K. S.; Väkeväinen, A. I.; Martikainen, J.-P.; Hakala, T. K.; Törmä, P. Ultrafast Pulse Generation in an Organic Nanoparticle-Array Laser. Nano Lett. 2018, 18, 2658-2665.

(25) Temnov, V. V.; Armelles, G.; Woggon, U.; Guzatov, D.; Cebollada, A.; Garcia-Martin, A.; Garcia-Martin, J.-M.; Thomay, T.; Leitenstorfer, A.; Bratschitsch, R. Active Magneto-Plasmonics in Hybrid Metal-Ferromagnet Structures. Nat. Photonics 2010, 4, 107111.

(26) Armelles, G.; Cebollada, G.; García-Martín, A.; González, M. U. Magnetoplasmonics: Combining Magnetic and Plasmonic Functionalities. Adv. Opt. Mater. 2013, 1, 10-35.

(27) Floess, D.; Giessen, H. Nonreciprocal Hybrid Magnetoplasmonics. Rep. Prog. Phys. 2018, 81, 116401.

(28) Maccaferri, N.; Gregorczyk, K. E.; de Oliveira, T. V. A. G.; Kataja, M.; van Dijken, S.; Pirzadeh, Z.; Dmitriev, A.; Åkerman, J.;
Knez, M.; Vavassori, P. Ultrasensitive and Label-Free Molecular-Level Detection Enabled by Light Phase Control in Magnetoplasmonic Nanoantennas. Nat. Commun. 2015, 6, 6150.

(29) Pourjamal, S.; Kataja, M.; Maccaferri, N.; Vavassori, P.; van Dijken, S. Hybrid $\mathrm{Ni} / \mathrm{SiO}_{2} / \mathrm{Au}$ Dimer Arrays for High-Resolution Refractive Index Sensing. Nanophotonics 2018, 7, 905-912.

(30) Zubritskaya, I.; Lodewijks, K.; Maccaferri, N.; Mekonnen, A.; Dumas, R. K.; Åkerman, J.; Vavassori, P.; Dmitriev, A. Active Magnetoplasmonic Ruler. Nano Lett. 2015, 15, 3204-3211.

(31) Kataja, M.; Freire-Fernández, F.; Witteveen, J.; Hakala, T.; Törmä, P.; van Dijken, S. Plasmon-Induced Demagnetization and Magnetic Switching in Nickel Nanoparticle Arrays. Appl. Phys. Lett. 2018, 112, No. 072406.

(32) Zou, S.; Schatz, G. C. Silver Nanoparticle Array Structures That Produce Giant Enhancements in Electromagnetic Fields. Chem. Phys. Lett. 2005, 403, 62-67.

(33) Kravets, V. G.; Schedin, F.; Grigorenko, A. N. Extremely Narrow Plasmon Resonances Based on Di_raction Coupling of Localized Plasmons in Arrays of Metallic Nanoparticles. Phys. Rev. Lett. 2008, 101, No. 087403.

(34) Auguié, B.; Barnes, W. L. Collective Resonances in Gold Nanoparticle Arrays. Phys. Rev. Lett. 2008, 101, 143902.

(35) Humphrey, A. D.; Barnes, W. L. Plasmonic Surface Lattice Resonances on Arrays of Different Lattice Symmetry. Phys. Rev. B: Condens. Matter Mater. Phys. 2014, 90, No. 075404.

(36) Wang, W.; Ramezani, M.; Väkeväinen, A. I.; Törmä, P.; Gómez Rivas, J.; Odom, T. W. The Rich Photonic World of Plasmonic Nanoparticle Arrays. Mater. Today 2018, 21, 303-314.

(37) Kravets, V. G.; Kabashin, A. V.; Barnes, W. L.; Grigorenko, A. N. Plasmonic Surface Lattice Resonances: A Review of Properties and Applications. Chem. Rev. 2018, 118, 5912-5951.

(38) Hakala, T. K.; Moilanen, A. J.; Väkeväinen, A. I.; Guo, R.; Martikainen, J.-P.; Daskalakis, K. S.; Rekola, H. T.; Julku, A.; Törmä, P. Bose-Einstein Condensation in a Plasmonic Lattice. Nat. Phys. 2018, 14, 739-744.

(39) Kataja, M.; Hakala, T. K.; Julku, A.; Huttunen, M. J.; van Dijken, S.; Törmä, P. Surface Lattice Resonances and MagnetoOptical Response in Magnetic Nanoparticle Arrays. Nat. Commun. 2015, 6, 7072.

(40) Maccaferri, N.; Bergamini, L.; Pancaldi, M.; Schmidt, M. K.; Kataja, M.; van Dijken, S.; Zabala, N.; Aizpurua, J.; Vavassori, P. Anisotropic Nanoantenna-Based Magnetoplasmonic Crystals for Highly Enhanced and Tunable Magneto-Optical Activity. Nano Lett. 2016, 16, 2533-2542.

(41) Guo, R.; Necada, M.; Hakala, T. K.; Väkeväinen, A. I.; Törmä, P. Lasing at K Points of a Honeycomb Plasmonic Lattice. Phys. Rev. Lett. 2019, 122, No. 013901.

(42) Bahari, B.; Ndao, A.; Vallini, F.; El Amili, A.; Fainman, Y.; Kanté, B. Nonreciprocal Lasing in Topological Cavities of Arbitrary Geometries. Science 2017, 358, 636-640.

(43) Harari, G.; Bandres, M. A.; Lumer, Y.; Rechtsman, M. C.; Chong, Y. D.; Khajavikhan, M.; Christodoulides, D. N.; Segev, M. Topological Insulator Laser: Theory. Science 2018, 359, eaar4003.

(44) Bandres, M. A.; Wittek, S.; Harari, G.; Parto, M.; Ren, J.; Segev, M.; Christodoulides, D. N.; Khajavikhan, M. Topological Insulator Laser: Experiments. Science 2018, 359, eaar4005.

(45) Khanikaev, A. B.; Shvets, G. Two-Dimensional Topological Photonics. Nat. Photonics 2017, 11, 763-773.

(46) Haldane, F. D. M.; Raghu, S. Possible Realization of Directional Optical Waveguides in Photonic Crystals with Broken Time-Reversal Symmetry. Phys. Rev. Lett. 2008, 100, No. 013904.

(47) Lu, L.; Joannopoulos, J. D.; Soljacic, M. Topological States in Photonic Systems. Nat. Phys. 2016, 12, 626-629.

(48) Klembt, S.; Harder, T. H.; Egorov, O. A.; Winkler, K.; Ge, R.; Bandres, M. A.; Emmerling, M.; Worschech, L.; Liew, T. C. H.; Segev, M.; Schneider, C.; Höfling, S. Exciton-Polariton Topological Insulator. Nature 2018, 562, 552-556. 
(49) Kataja, M.; Pourjamal, S.; van Dijken, S. Magnetic Circular Dichroism of Non-Local Surface Lattice Resonances in Magnetic Nanoparticle Arrays. Opt. Express 2016, 24, 3562-3571.

(50) Dresselhaus, M. S.; Dresselhaus, G.; Jorio, A. Group Theory: Application to the Physics of Condensed Matter; Springer-Verlag: Berlin, 2008.

(51) Bradley, C. J.; Cracknell, A. P. The Mathematical Theory of Symmetry in Solids; Representation Theory for Point Groups and Space Groups; Clarendon Press: Oxford, 1972.

(52) Johnson, P. B.; Christy, R. W. Optical Constants of Transition Metals: Ti, V, Cr, Mn, Fe, Co, Ni, and Pd. Phys. Rev. B 1974, 9, 5056-5070. 